\title{
Vitreous concentrations of vascular endothelial growth factor as a potential biomarker for postoperative complications following pars plana vitrectomy
}

Faruk Nisic ${ }^{1}$, Nina Jovanovic ${ }^{2}$, Milka Mavija ${ }^{3}$, Emina Alimanovic-Halilovic ${ }^{1}$, Aida Nisic ${ }^{4}$, Orhan Lepara ${ }^{5}$, Adem Cemerlic

\author{
${ }^{1}$ Clinic for Eye Disease, Clinical Centre University Sarajevo, Sarajevo, \\ Bosnia and Herzegovina \\ ${ }^{2}$ Ophthalmology Department, Canton Hospital Zenica, Zenica, \\ Bosnia and Herzegovina \\ ${ }^{3}$ Department of Ophthalmology at School of Medicine, University of Banja Luka; \\ University Clinical Centre of the Republic of Srpska, Banja Luka, Bosnia and \\ Herzegovina \\ ${ }^{4}$ Specialty Consultative Health Care of PI Health Centre of Sarajevo Canton, Sarajevo, \\ Bosnia and Herzegovina \\ ${ }^{5}$ Department of Human Physiology, School of Medicine, University of Sarajevo, \\ Sarajevo, Bosnia and Herzegovina \\ ${ }^{6}$ School of Medicine, University of Sarajevo, Sarajevo, Bosnia and Herzegovina
}

Submitted: 9 November 2017

Accepted: 29 December 2017

Arch Med Sci 2019; 15, 2: 449-456

DOI: https://doi.org/10.5114/aoms.2018.73208

Copyright @ 2018 Termedia \& Banach

\section{Abstract}

Introduction: The aim of the study was to investigate the effects of vascular endothelial growth factor (VEGF) concentration in vitreous on postoperative complications after pars plana vitrectomy (PPV).

Material and methods: Ninety subjects were surgically treated with PPV and followed up for 12 months at the Clinical Centre University Sarajevo, Clinic for Eye Disease. Exclusion criteria were presence of other eye diseases, systemic acute/chronic inflammatory conditions, or malignant neoplasms; previously performed PPV surgery; previously received intravitreal or systemic anti-VEGF therapy. A vitreous sample was obtained while performing the PPV procedure, using the Quantikine ELISA test to determine VEGF level, as a risk factor. Outcome measures were intraoperative and postoperative complications reported using categorical data: blunt and sharp dissection of membranes, intraoperative hemorrhage stopped by increasing infusion pressure, pressing with blunt instrument, or using diathermy. The following postoperative complications were assessed on the first day and at the 12-month follow-up visit: vitreous hemorrhage, fibrovascular proliferation (FVP), rubeosis iridis, and neovascular glaucoma (NVG).

Results: Levels of vitreous VEGF at the time of PPV were significantly higher in eyes with: vitreous hemorrhage on the first day after PPV $(p=0.003)$; FVP on the first day and 12 months after PPV $(p=0.002$ and $p<0.001$, respectively); iris rubeosis on the first day and 12 months after PPV surgery ( $p<0.001$, and $p=0.001$, respectively); NVG on the first day and 12 months after PPV surgery ( $p=0.043$ and $p=0.011$, respectively), compared to the eyes without complications.

Conclusions: Preoperative levels of VEGF in vitreous can be a useful biomarker and predictor of the postoperative outcome in terms of intraoperative and postoperative complications.

Key words: vascular endothelial growth factor, pars plana vitrectomy, proliferative diabetic retinopathy, postoperative complications, biomarker.

\author{
Corresponding author: \\ Nina Jovanovic MD \\ Ophthalmology Department \\ Canton Hospital Zenica \\ 67 Crkvice St \\ 72000 Zenica \\ Bosnia and Herzegovina \\ Phone: +38761276422 \\ E-mail: nina-jovanovic@ \\ uiowa.edu
}




\section{Introduction}

Pars plana vitrectomy (PPV) is a microsurgical procedure used for treating different retinal diseases. One of those is proliferative diabetic retinopathy (PDR) when laser photocoagulation or pharmacological therapy does not provide positive results, or where a mechanical component is evident [1]. Due to the disease's complexity, and the intricacy of the PPV operation, the surgeon often encounters various complications, which may seldom be predictable and may appear intra- and postoperatively [2]. Pars plana vitrectomy at times may have noteworthy intraoperative complications. Examples include intraretinal or subretinal hemorrhage, retinal tear, retinectomy and retinotomy [3]. These complications may be categorized into two groups: 1 . lacerations of conjunctiva, corneal edema, cataract and iris trauma, which are individual and most often dependent on the operative techniques, and 2. glaucoma, intraoperative and postoperative retinal hemorrhage, peripheral retinal tear, gliosis, recurrence of fibrous tissue proliferation, and proliferation of fibrovascular tissue at sclerotomy sites [4].

Vascular endothelial growth factor (VEGF) is the most potent vasoactive factor, which induces angiogenesis and increases vascular permeability [5]. Possessing a central role in the processes of angiogenesis, VEGF signals a cascade which is significant for numerous cellular events, such as endothelial cell proliferation and migration, remodeling of the extracellular matrix, increased vascular permeability and the survival of newly formed blood vessels [6]. There are numerous pathological conditions and diseases where VEGF plays a very important role in the pathogenesis, whereby ischemic neovascular eye diseases are distinguished. Within the VEGF family, VEGF-A plays a pivotal role, due to its strong potency for angiogenesis stimulation [7]. It has been proven that in many diseases the intraocular level of VEGF-A is correlated with the formation of new blood vessels $[8,9]$. Through numerous inflammatory mediations VEGF is involved in the processes of chemotaxis, inflammation, and neuroprotection. In cases when the blood-retinal barrier is disrupted, the role of VEGF is emphasized, since it has 50000 times higher potency in induction of vascular permeability than histamine. Previous studies have shown that the level of VEGF in the vitreous or aqueous humor was significantly higher in patients who had postoperative complications such as vitreous hemorrhage (VH), or neovascular glaucoma (NVG) in comparison with those who did not have such complications after PPV [10]. The aim of this study was to investigate whether vitreous concentrations of VEGF may be used as a potential biomarker and predict postoperative complications of PPV.

\section{Material and methods}

\section{Patients}

The research was designed as a clinical prospective, longitudinal, cohort study, conducted at the Clinical Centre University of Sarajevo, Clinic for Eye Diseases and Clinic for Immunology. The study was conducted on a sample of 90 subjects (90 eyes) in the period of 2014 to 2016 . It included both male and female patients older than 18 , in whom PPV surgery was conducted upon presence of a relevant indication. Treated patients had diagnosed proliferative diabetic retinopathy (PDR), which was the primary indication for PPV treatment, or PPV treatment was indicated due to reasons other than PDR (rhegmatogenous retinal detachment, vitreoretinopathy, macular hole, and eye trauma). The inclusion criterion was signed informed consent, where they signed a statement that the results obtained during treatment could be used for the purposes of research in accordance with the principles of the Helsinki Declaration. Exclusion criteria were presence of other eye diseases: glaucoma, uveitis, central retinal artery occlusion (CRAO), or central retinal vein occlusion (CRVO); presence of systemic acute, or chronic inflammatory conditions, or malignant neoplasms; previously performed PPV surgery; previously received intravitreal or systemic anti-VEGF therapy; and disorientation at presentation.

\section{Study variables and clinical data analysis}

In all subjects, preoperative baseline data were obtained and included in the study. Data on the following categorical and continuous variables were obtained: initials, sex, age, treatment duration, type and duration of diabetes mellitus, comorbidity with hypertension, or other illnesses during the period of inclusion in the study. The following ophthalmological tests were performed for all subjects: best corrected visual acuity (BCVA) using a Snellen chart, intraocular pressure (IOP) using Goldman applanation tonometry, slit-lamp examination of anterior and posterior eye segment (with 90D, double aspheric lens), ultrasound of the eye with $A$ and $B$ scan, optical coherence tomography (OCT) and continuous data for laboratory blood testing as part of the preoperative preparation (differential blood count, hemoglobin, sedimentation rate, hematocrit, electrolytes, glucose level, urea, creatinine, and $\mathrm{HbA}_{1 c}$ ). Intraoperatively, categorical variables were used to depict: types of surgery, intraoperative use of endolaser photocoagulation and internal tamponade of retina with air, expanding gas sulfur hexafluoride (SF6), octafluoropropane (C3F8), or silicone oil. Using continuous data levels of VEGF were measured as a risk factor for occurrence of intraoper- 
ative and postoperative complications. Outcome measures were intraoperative and postoperative complications reported using categorical data. Reported intraoperative complications included: blunt dissection of the membranes, sharp membrane dissection, intraoperative hemorrhage stopped by increasing infusion pressure (IP), or by pressing with a blunt instrument, and intraoperative bleeding stopped using diathermy. Postoperative complications were assessed on the first day and at the $12^{\text {th }}$ month follow-up. Data were retested, recorded and categorized as early and late complications: $\mathrm{VH}$, fibrovascular proliferation (FVP), iris rubeosis (IR), and secondary glaucoma.

\section{Surgical technique}

After obtaining informed consent, ophthalmological testing and diagnostic procedures, followed by PPV surgery, were performed. Pars plana vitrectomy procedures were done using a standard 20 G until April 2015. After April 2015, 23 G three-port PPV procedures in local potentiated retrobulbar anaesthesia, or in general endotracheal anaesthesia (ETA), were performed. Vitreous gel was operatively removed using necessary surgical manipulations (removal of pathological structures: proliferation, membrane, blood, foreign bodies, etc.). Depending on the lens status, combined phacoemulsification (PHACO) and the implantation of an acrylate intraocular lens (IOL) were performed. In some cases, removal of the internal limiting membrane (ILM) in addition to the use of retinal dye, as well as necessary retinotomy in cases of marked retinal contraction combined with proliferative vitreoretinopathy (PVR), were further performed. Optical coherence tomography examination was used for the purpose of evaluating indications for PPV surgery and pre- and postoperative results when the optic media was not clear and no other method could be used for the evaluation. According to the surgical protocol and clinical findings, we conducted selective endolaser-photocoagulation and internal retinal tamponade with air, balanced salt solution (BSS), SF6, C3F8, or silicone oil.

\section{Sample collection and measurements of VEGF}

A vitreous (CV) sample was obtained while performing PPV surgery. The sample was taken in sterile conditions, using a special tank (silicone tube) of $1 \mathrm{~cm}$ volume, which was originally designed for purpose of this research, and placed in the aspiration line of the vitrectomy between the vitrectomy probe and cassette for the collection of fluids, which were surgically removed from the eye. 0.51.0 cc of undiluted CV was aspirated before each opening of the infusion line. The sample of CV was centrifuged, and stored in a refrigerator at $-80^{\circ} \mathrm{C}$. Levels of VEGF in the CV were determined by an enzyme-linked immunosorbent assay (ELISA). All procedures were performed in the Immunology Clinic, Clinical Centre of the University of Sarajevo. The quantitative values of VEGF concentration in $C V$ were recorded and used in the statistical analysis. To determine value of VEGF in CV, the Quantikine ELISA Kit (code number: DVEOO, SVEOO and PDVEO0) was used.

\section{Statistical analysis}

Statistical analysis was performed using IBM SPSS Statistics version 21.0. The results were expressed as the median and interquartile range $\left(25^{\text {th }}-75^{\text {th }}\right.$ percentiles). To test significant difference in deviation from the normal distribution, the Kolmogorov-Smirnov test was performed. Results were analyzed by non-parametric tests (Kruskal-Wallis test and Mann-Whitney $U$ test), assuming the distribution deviated from normal. For dependent variables that did not follow a normal distribution, the Wilcoxon test was used. In the analysis of the dependence between categorical variables, Fisher's exact test was performed, at $\alpha=0.05$. Specificity and sensitivity of potential disease markers and the incidence of PPV complications were examined by the ROC curve.

\section{Results}

Of the total of 90 surgically treated patients, a slight majority were male: $57.8 \%$. Slit-lamp results were pathological in $25.6 \%$ of patients. Most common clinical findings diagnosed by the ultrasound were retinal detachment in $51.1 \%$ of cases, followed by hemophthalmos + PDR in $27.8 \%$, and the least frequent was hemophthalmos in only $21.1 \%$ of cases. PPV was performed in $53.3 \%$, PPV $+\mathrm{PHACO}$ in $43.3 \%$, while other procedures (lensectomy for example) were performed in $3.3 \%$. Surgical complications were reported in $25.6 \%$ of patients, with blunt dissection being performed in $60 \%$ and sharp dissection in $35.6 \%$ of cases. Irrigation pressure elevation was applied in $11.1 \%$, diathermy in $14.4 \%$ of cases. Preoperative laser photocoagulation (LPC) was performed in 33.3\% of patients. The most frequent was silicon tamponade in $48.9 \%$ of cases (Table I).

Frequency distribution comparing postoperative complications on the first day and 12 months after surgery was tested. The most frequently reported complication was FVP in $57.8 \%$ of cases, followed by fibrin exudation (FE), and VH in $44.3 \%$ and $40.0 \%$, respectively. Among early postoperative complications, FE and $\mathrm{VH}$ were most common (37.0\% and $24.0 \%$, respectively), while retinal 
Table I. Frequency distribution of patient characteristics, baseline ocular findings, surgical methods and outcomes

\begin{tabular}{|c|c|c|}
\hline Variables & $\begin{array}{c}\text { Total } \\
\text { number }(N)\end{array}$ & $\begin{array}{c}\text { Percentage } \\
(\%)\end{array}$ \\
\hline Gender: & 90 & 100.0 \\
\hline Male & 52 & 57.8 \\
\hline Female & 38 & 42.2 \\
\hline Slit-lamp results: & 90 & 100.0 \\
\hline Normal & 67 & 74.4 \\
\hline Pathological & 23 & 25.6 \\
\hline Surgical procedure: & 90 & 100.0 \\
\hline PPV & 48 & 53.3 \\
\hline $\mathrm{PPV}+\mathrm{PHACO}$ & 39 & 43.3 \\
\hline Other & 3 & 3.3 \\
\hline Ultrasound results: & 90 & 100.0 \\
\hline Hemophthalmos & 19 & 21.1 \\
\hline Hemophthalmos + PDR & 25 & 27.8 \\
\hline Retinal detachment & 46 & 51.1 \\
\hline Operative complications: & 90 & 100.0 \\
\hline Yes & 23 & 25.6 \\
\hline No & 67 & 74.4 \\
\hline Blunt dissection: & 90 & 100.0 \\
\hline Yes & 54 & 60.0 \\
\hline No & 36 & 40.0 \\
\hline Sharp dissection: & 90 & 100.0 \\
\hline Yes & 32 & 35.6 \\
\hline No & 58 & 64.4 \\
\hline IP: & 90 & 100.0 \\
\hline Yes & 10 & 11.1 \\
\hline No & 80 & 88.9 \\
\hline Diathermy: & 90 & 100.0 \\
\hline Yes & 13 & 14.4 \\
\hline No & 77 & 85.6 \\
\hline LPC: & 90 & 100.0 \\
\hline Yes & 30 & 33.3 \\
\hline No & 60 & 66.7 \\
\hline Tamponade: & 90 & 100.0 \\
\hline BSS air & 7 & 7.8 \\
\hline SF6, C3F8 & 39 & 43.3 \\
\hline Silicone & 44 & 48.9 \\
\hline
\end{tabular}

PPV - pars plana vitrectomy, PDR - proliferative diabetic retinopathy, IP - bleeding stopped by increasing infusion, LPC laser photocoagulation. detachment (RD) and suprachoroid hemorrhage were the least common $(1.0 \%$ and $3.0 \%$, respectively). Among late postoperative complications, FVP and IR were most common (34.0\% and 19.0\%, respectively), while FE and suprachoroid hemorrhage were least common (3.0\% both) (Table II).

The median preoperative IOP value was $16 \mathrm{~mm} \mathrm{Hg}(14-18)]$, and was significantly lower than IOP 12 months after surgery $(18 ; 15-22$; $p=0.001)$. The median preoperative BCVA value was $0.001(0.001-0.003)$ and was significantly lower than median BCVA 12 months after surgery: $0.3(0.004-0.4)(p<0.001)$, Table III.

Levels of VEGF at the time of vitrectomy in the vitreous were significantly higher in eyes with $\mathrm{VH}$ on the first day after PPV than in those without early VH $(p=0.003)$. Levels of VEGF at the time of vitrectomy in the vitreous were significantly higher in eyes with FVP on the first day and 12 months after PPV than in eyes without FVP, $p=0.002$ and $p<0.001$, respectively. Levels of VEGF at the time of vitrectomy in the vitreous were significantly higher in eyes with IR on the first day after PPV and 12 months after surgery compared with the eyes without complications, $p<0.001$, and $p=$ 0.001 , respectively, and in eyes with NVG 1 day after PPV and 12 months after PPV, $p=0.043$ and $p=0.011$, respectively, compared with the eyes without complications (Table IV).

Vascular endothelial growth factor sensitivity for $\mathrm{VH}$ on the first day after PPV was $75 \%$ and specificity $63.6 \%$. Vascular endothelial growth factor sensitivity for FVP on the first day and 12 months after PPV was $66.6 \%$ and $76.5 \%$, respectively, while specificity was $81.8 \%$ and $67.8 \%$, respectively. Vascular endothelial growth factor sensitivity for IR on the first postoperative day and 12 months after PPV was $91.6 \%$ and $84.2 \%$, respectively, and specificity was $79.4 \%$ and $66.1 \%$, respectively. Vascular endothelial growth factor sensitivity for NVG on the first day after PPV was $66.6 \%$ and specificity $75.6 \%$, while at 12 months after PPV sensitivity was $59.0 \%$ and specificity 79.4\% (Table V).

\section{Discussion}

This was a prospective, longitudinal cohort study of surgically treated patients with PPV where levels of VEGF were preoperatively measured and postoperative complications evaluated. Our findings support the hypothesis that an increased level of VEGF is associated with early and late postoperative complications following PPV surgery. The study by Funatsu et al. [11] found that preoperative VEGF levels correlate with the success of PPV surgery. Our results suggest that preoperative measurement of the VEGF level can be used as a biological indicator for the surgery results. 
Table II. Frequency distribution of postoperative complications reported 1 day postoperatively and 12 months after surgery

\begin{tabular}{|c|c|c|c|c|c|c|c|}
\hline \multirow[t]{2}{*}{ Postoperative complications } & \multicolumn{2}{|c|}{ Total } & \multicolumn{2}{|c|}{$\begin{array}{l}\text { Post-operative } \\
\text { control } 1^{\text {st }} \text { day }\end{array}$} & \multicolumn{2}{|c|}{$\begin{array}{l}\text { Post-operative } \\
\text { control } 12^{\text {th }} \text { month }\end{array}$} & \multirow[t]{2}{*}{$P$-value* } \\
\hline & $N$ & $\%$ & $N$ & $\%$ & $N$ & $\%$ & \\
\hline Vitreous hemorrhage & 36 & 40.0 & 24 & 26.7 & 12 & 13.3 & 0.002 \\
\hline Fibrovascular proliferation & 52 & 57.8 & 18 & 20.0 & 34 & 37.8 & $<0.001$ \\
\hline Fibrin exudation & 40 & 44.3 & 37 & 41.1 & 3 & 3.3 & - \\
\hline Iris rubeosis & 31 & 34.4 & 12 & 13.3 & 19 & 21.1 & $<0.001$ \\
\hline Retinal detachment & 8 & 8.9 & 1 & 1.1 & 7 & 7.8 & - \\
\hline Suprachoroid hemorrhage & 6 & 6.6 & 3 & 3.3 & 3 & 3.3 & - \\
\hline Neovascular glaucoma & 34 & 37.7 & 12 & 13.3 & 22 & 24.4 & $<0.001$ \\
\hline
\end{tabular}

${ }^{\star}$ Fisher's exact test for testing independence $(\alpha=0.05)$.

Table III. Value of intraocular pressure and best corrected visual acuity preoperatively and 12 months after surgery

\begin{tabular}{|lccccc|}
\hline Variable & \multicolumn{2}{c}{ Preoperative } & 12 months postoperatively & \multirow{2}{*}{-value* $^{*}$} \\
\cline { 2 - 5 } & Median & $\begin{array}{c}25^{\text {th }}-75^{\text {th }} \\
\text { percentile }\end{array}$ & Median & $\begin{array}{c}25^{\text {th }}-75^{\text {th }} \\
\text { percentile }\end{array}$ & \\
\hline Intraocular pressure $[\mathrm{mm} \mathrm{Hg}]$ & 16 & $14-18$ & 18 & $15-22$ & 0.001 \\
\hline Best corrected visual acuity & 0.001 & $0.001-0.03$ & 0.3 & $0.004-0.4$ & $<0.001$ \\
\hline
\end{tabular}

${ }^{\star}$ Fisher's exact test at $\alpha=0.05$.

Table IV. Levels of VEGF at the time of primary vitrectomy and postoperative complications

\begin{tabular}{|c|c|c|c|c|c|}
\hline \multirow{3}{*}{$\begin{array}{l}\text { Variables } \\
\begin{array}{l}\text { Vitreous hemorrhage } \\
1^{\text {st }} \text { day after PPV** }\end{array}\end{array}$} & \multicolumn{4}{|c|}{ VEGF $[\mathrm{pg} / \mathrm{ml}]$} & \multirow{3}{*}{$\begin{array}{r}P \text {-value* } \\
0.003\end{array}$} \\
\hline & \multicolumn{2}{|c|}{ With complications } & \multicolumn{2}{|c|}{ Without complications } & \\
\hline & 448.66 & $83.34-117.80$ & 48.62 & $2121-378.42$ & \\
\hline \multicolumn{6}{|c|}{ Fibrovascular proliferation: } \\
\hline 1 day after PPV & 926.35 & $82.86-1072.34$ & 52.98 & $21.76-358.12$ & 0.002 \\
\hline 12 months after PPV & 445.44 & 84.59-1020.94 & 39.77 & $20.67-269.31$ & $<0.001$ \\
\hline \multicolumn{6}{|l|}{ Iris rubeosis: } \\
\hline 1 day after PPV & 972.96 & $757.77-1146.96$ & 57.34 & $21.21-331.86$ & $<0.001$ \\
\hline 12 months after PPV & 903.10 & 251.94-1140.53 & 48.62 & $21.40-318.76$ & 0.001 \\
\hline \multicolumn{6}{|l|}{ Neovascular glaucoma: } \\
\hline 1 day after PPV & 833.14 & $53.71-1124.12$ & 69.03 & $22.50-439.15$ & 0.043 \\
\hline 12 months after PPV & 611.95 & $32.60-1142.67$ & 57.34 & $21.76-358.12$ & 0.011 \\
\hline
\end{tabular}

*Mann-Whitney $U$ test at $\alpha=0.05 .{ }^{* *}$ Vitreous hemorrhage 12 months after was insignificant and omitted from the Table.

Evidence of the risk factors associated with an early VH following PPV are scarce. The most often reported risk factors are age and severity of diabetic retinopathy [12]. Recently, studies by Wakabayashi et al. [10], Funatsu et al. [11], and Yan et al. [13] added increased VEGF levels as a risk factor in the body of the literature. Our study did not reveal any significant association between levels of VEGF and late $\mathrm{VH}$; however, an association was found in the case of early $\mathrm{VH}$. This finding concurs with the aforementioned studies. The result is supported by Wakabayashi et al., who reported an odds ratio of 5.1 for sustaining an early $\mathrm{VH}$ when there is an increased level of VEGF in the vitreous. Their results suggest that high vitreous levels of VEGF prior to vitrectomy have high neovascular activity and may cause extensive proliferative changes; therefore, such subjects may be more susceptible to the development of postoperative VH. However, the exact mechanism that could explain the 
Table V. Sensitivity and specificity of VEGF in vitreous as a marker of the occurrence of postoperative complications of PPV

\begin{tabular}{|llcc|}
\hline Variables & AUC & Sensitivity (\%) & Specificity (\%) \\
\hline Vitreous hemorrhage 1 day after PPV & 0.708 & 75.0 & 63.6 \\
\hline \begin{tabular}{l} 
Fibrovascular proliferation: \\
\hline 1 day after PPV
\end{tabular} & 0.736 & 66.6 & 81.8 \\
\hline 12 months after PPV & 0.721 & 76.5 & 67.8 \\
\hline \begin{tabular}{l} 
Rubeosis iridis: \\
\hline 1 day after PPV
\end{tabular} & 0.838 & 91.6 & 79.4 \\
\hline 12 months after PPV & 0.758 & 84.2 & 66.1 \\
\hline Neovascular glaucoma: & & & 75.6 \\
\hline 1 day after PPV & 0.682 & 66.6 & 79.4 \\
\hline 12 months after PPV & 0.680 & 59.0 & \\
\hline
\end{tabular}

association of VEGF and the onset $\mathrm{VH}$ is still not sufficiently illuminated. Often, surgery with intraoperative hemorrhage shows an increased risk for postoperative bleeding. A number of researchers believe that intravitreal application of bevacizumab (IVB) is useful for avoiding further hemorrhage [14]. Accordingly, meta-analytical studies state that the use of preoperative or intraoperative IVB reduces the incidence of early vitreous hemorrhage. Randomized studies that look at other anti-VEGF drugs are ongoing and will strengthen the current results, giving surgeons and respondents evidence that will lead to the choice of PDR treatment [15]. The same study reported an association between IVB and incidence of $\mathrm{VH}$ reduction, suggesting an important role of VEGF in post-PPV surgery complications.

Numerous studies have shown an association between levels of VEGF and FVP [16], which concurs with our study results. Similarly as with $\mathrm{VH}$, previous studies have shown that during the use of anti-VEGF drugs, the benefit of these is reflected in the occurrence of active fibrovascular tissue regression [17]. $\mathrm{RI}$ is a serious complication especially in diabetic retinopathy and CRVO. It was observed in our study as an early and late postoperative complication and associated with increased vitreous levels of VEGF $(903.10 \mathrm{pg} / \mathrm{ml}$ in patients who show RI 12 months postoperatively compared to $48.62 \mathrm{pg} / \mathrm{ml}$ in patients without late $\mathrm{RI} ; p=0.001)$. Similar results $(p<0.001)$ are observed for early RI with a significant difference in VEGF levels. Cytokines, a particular vascular growth factor, are responsible for the formation of new blood vessels. Our study showed a significant association between early and late NVG and levels of VEGF, higher levels being found in patients with postoperative NVG than those without NVG.
A study by Wakabayashi et al. reported a significant association of postoperative NVG and increased levels of VEGF, which is in line with our results [10]. The results of their research suggest that a high intraocular level of VEGF may be the most important factor associated with the occurrence of postoperative glaucoma [10].

Antiangiogenic treatment that inhibits the effects of VEGF and its receptors offers numerous possibilities in the treatment of neovascular diseases [18]. Thus, Tu et al. [19] have shown that multiple intravitreal injections of ranibizumab can be a safe, effective supplemental therapy for PDR and diabetic RI. Salman [20] concludes that intra-silicon injection of bevacizumab is safe and effective in treating neovascularization of the iris after vitrectomy in patients with advanced PDR. Increasing the number of receptors for VEGF in the eye does not limit ischemic retinal disorder. Research has shown contradictory results regarding the intravitreal use of anti-VEGF drugs with the goal of preventing NVG. A number of researchers believe that intravitreal use of anti-VEGF may improve clinical manifestation of NVG and that treatment with these drugs could be very useful [21]. There have been published controversial studies in which other researchers disagree with the aforementioned view, believing that the anti-VEGF application itself, and especially its longer use, can lead to an increase in IOP, thus increasing the risk of glaucoma occurrence and progression [22].

In this study, we propose the use of vitreous level of VEGF as a biomarker for prognosis of surgical complications following PPV. Biomarkers' predictive role can be widely utilized as a cost-saving and beneficial method for disease prediction and possible prevention. An exam- 
ple of this is the study by Sayan and Kotan [23] suggesting predictive use of the plasma levels of brain natriuretic peptide as a useful biomarker to diagnose and predict the severity of acute ischemic stroke. An additional advantage of their study's biomarker is that it could be applied as a predictor of mortality and morbidity even in patients without an ischemic episode. A study by Das [24] suggests that measurement of plasma and vitreous levels of VEGF can be used to correlate concentration of VEGF with staging of diabetic retinopathy, diabetic macular edema and age-related macular degeneration. In the case of VEGF level, factors such as environment, genetics and endocrine status may vary individually and influence levels of VEGF independently from diabetic retinopathy [25]. This may influence the effect of the association between levels of VEGF and postoperative complications following PPV surgery in our study. However, these individual VEGF variation levels are probably equally distributed among patients; therefore, the error is non-differential and does not affect the validity of the study results.

Our study has several limitations. Although the sample size is relatively large, a study with a larger sample could improve the statistical power. Additionally, a sample of subjects who received preoperatively anti-VEGF treatment included in the comparative study could have given a clinically more significant result and evidence of what was indirectly shown with our study - benefits of intravitreal anti-VEGF application for preventing complications following PPV. A larger, perhaps multi-centric study with all three subject groups would be beneficial in the future.

An important methodological strength of this study is its prospective, longitudinal cohort design, and relatively large sample size of surgically treated subjects. The results of the study added to the evidence level of benefits of anti-VEGF in the preoperative treatment. The predictive role of VEGF concentration levels in the human eye opens new treatment possibilities in terms of preoperative preparation with the goal of its VEGF level decrease.

In conclusion, this study contributes to the level of evidence that VEGF vitreous level is associated with the occurrence of postsurgical complication following PPV. Although several studies have already reported significant ORs and an association for late VH, FVP or retinal detachment following PPV, our study revealed a significant association between increased levels of VEGF and late VH, FVP, $\mathrm{RI}$ and NVG. This suggests that preoperative measurement of VEGF plasma levels and IVB injection when indicated may reduce the incidence of intraand postoperative complications following PPV.

\section{Conflict of interest}

The authors declare no conflict of interest.

\section{References}

1. Lai FH, Lo EC, Chan VC, Brelen M, Lo WL, Young AL. Combined pars plana vitrectomy-scleral buckle versus pars plana vitrectomy for proliferative vitreoretinopathy. Int Ophthalmol 2016; 36: 217-24.

2. Abouammoh MA, Abouammoh MA, Gale JS, Arevalo JF, Sharma S. A novel technique for securing sclerotomies in 20-gauge transconjunctival pars plana vitrectomy: surgical outcomes and complications in 529 consecutive cases. Retina 2016; 36: 974-80.

3. Chao D, Gonzalez M, Flynn H. Giant retinal tears after pars plana vitrectomy for diabetic vitreous hemorrhage. Retin Physician 2012; 9: 40-2.

4. Steve C. Complications of diabetic vitreoretinal surgery. Retin Physician 2010; 7: 41-6.

5. Canavese M, Spaccapelo R. Protective or pathogenic effects of vascular endothelial growth factor (VEGF) as potential biomarker in cerebral malaria. Pathog Glob Health 2014; 108: 67-75.

6. Mehta LJ, Dhalla NS. Biochemical Basis and Therapeutic Implications of Angiogenesis. Springer International Publishing, New York 2013.

7. Ferrara N. Role of vascular endothelial growth factor in physiologic and pathologic angiogenesis: therapeutic implications. Semin Oncol 2002; 29: 10-4.

8. Fogli S, Mogavero S, Egan CG, Del Re M, Danesi R. Pathophysiology and pharmacological targets of VEGF in diabetic macular edema. Pharmacol Res 2016; 103: 149-57.

9. Amadio M, Govoni S, Pascale A. Targeting VEGF in eye neovascularization: What's new? A comprehensive review on current therapies and oligonucleotide-based interventions under development. Pharmacol Res 2016; 103: 253-69.

10. Wakabayashi Y, Usui Y, Okunuki Y, et al. Intraocular VEGF level as a risk factor for postoperative complications after vitrectomy for proliferative diabetic retinopathy. Invest Ophthalmol Vis Sci 2012; 53: 6403-10.

11. Funatsu H, Yamashita H, Miura T, Noma H, Nakamura S, Hori S. Risk evaluation of outcome of vitreous surgery based on vitreous levels of cytokines. Eye 2007; 21: 377-82.

12. Yang $\mathrm{CM}$, Yeh PT, Yang $\mathrm{CH}$. Intravitreal long-acting gas in the prevention of early postoperative vitreous hemorrhage in diabetic vitrectomy. Ophthalmology 2007; 114: 710-5.

13. Yan H, Cui J, Yu JG, et al. The expression of vascular endothelial growth factor of vitreous in patients with proliferative diabetic retinopathy. Zhonghua Yan Ke Za Zhi 2009; 45: 206-9.

14. Smith JM, Steel DHW. Anti-vascular endothelial growth factor for prevention of postoperative vitreous cavity hemorrhage after vitrectomy for proliferative diabetic retinopathy. Cochrane Database Syst Rev 2011; 5: CD008214.

15. Oshima Y, Shima C, Wakabayashi T, et al. Microincision vitrectomy surgery and intravitreal bevacizumab as a surgical adjunct to treat diabetic traction retinal detachment. Ophthalmology 2009; 116: 927-38.

16. Wirostko B, Wong TY, Simo R. Vascular endothelial growth factor and diabetic complications. Prog Retin Eye Res 2008; 27: 608-21. 
17. Moradian S, Ahmadieh H, Malihi M, Soheilian M, Dehghan MH, Azarmina M. Intravitreal bevacizumab in active progressive proliferative diabetic retinopathy. Graefe's Arch Clin Exp Ophthalmol 2008; 246: 1699-705.

18. Carrasco E, Garrido JM, Álvarez PJ, et al. Meroxest improves the prognosis of immunocompetent C57BL/6 mice with allografts of E0771 mouse breast tumor cells. Arch Med Sci 2016; 12: 919-27.

19. Tu Y, Fay C, Guo S, Zarbin MA, Marcus E, Bhagat N. Ranibizumab in patients with dense cataract and proliferative diabetic retinopathy with rubeosis. Oman J Ophthalmol 2012; 5: 161-5.

20. Salman AG. Intrasilicone bevacizumab injection for iris neovascularization after vitrectomy for proliferative diabetic retinopathy. Ophthalmic Res 2013; 49: 20-4.

21. Shen $X$, Chen Y, Wang Y, Yang L, Zhong Y. Intravitreal ranibizumab injection as an adjuvant in the treatment of neovascular glaucoma accompanied by vitreous hemorrhage after diabetic vitrectomy. J Ophthalmol 2016; 2016: 4108490

22. Lanzl I, Kotliar K. Can anti-VEGF injections cause glaucoma or ocular hypertension? Klin Monbl Augenheilkd 2017; 234: 191-3.

23. Sayan S, Kotan D. Levels of brain natriuretic peptide as a marker for the diagnosis and prognosis of acute ischemic stroke. Arch Med Sci Atheroscler Dis 2016; 1: e16-22.

24. Das UN. Diabetic macular edema, retinopathy and age-related macular degeneration as inflammatory conditions. Arch Med Sci 2016; 12: 1142-57.

25. Senel K, Baykal T, Seferoglu B, et al. Circulating vascular endothelial growth factor concentrations in patients with postmenopausal osteoporosis. Arch Med Sci 2013 9: 709-12. 